\section{Absence of temporal discrimination following septal lesions*}

\author{
PAUL ELLEN and WILLIAM C. AITKEN, JR \\ Georgia State University, Atlanta, Ga. 30303
}

Septally lesioned and normal rats were trained on a DRL 20 schedule, utilizing an IBM-1800 data acquisition and control system to program the reinforcement contingency and record responses. Normal animals developed a temporal discrimination (as measured by the IRTs/opportunity statistic), while septals did not. Training did not lead to any change in the frequency of reinforcements obtained by either group. These results were discussed in terms of differences in the locus of stimulation (i.e., internal or external) controlling performance.

Previous studies (Ellen, Wilson, \& Powell, $1964 ;$ M a Dougall, VanHoesen, \& Mitchell, 1969) have shown that, when interresponse times (IRTs) of septal animals barpressing on a DRL (differential reinforcement of low rates) schedule are converted to IRTs/opportunity (Anger, 1956), response probability increases as a function of the delay since the preceding response. The IRTs/OP is defined as the number of times an animal made a given delay divided by the number of occasions the animal actually delayed that long or longer, and is an unbiased estimate of response probability for equal IRT categories. Since each time the animal makes a given delay the opportunity for a longer delay is precluded, the IRT/OP statistic takes into account the opportunities for each given IRT that actually exist. The observation of an increasing response probability with increasing delays led to the conclusion that temporal discrimination is not impaired in septal animals.

Recently we have had occasion to run septal animals utilizing an IBM-1800 Data Acquisition and Control System, with the animals being isolated more than $200 \mathrm{ft}$ from the computer. As a result, there was no opportunity for any external noises associated with the programming and recording equipment to become correlated with the elapsing of the required delay and thus become an external anchor for the temporal discrimination. The present study is a report of our findings and is of particular interest because of the marked differences in results obtained as compared with those usually found when electromechanical or solid-state programming and recording methods are used.

\section{METHOD}

Twenty Long-Evans male hooded rats served as $\mathrm{Ss}$ in this study. The animals were about 120 days of age

* This research supported in part by NSF Grant GB-7184. and weighed approximately $300 \mathrm{~g}$ at the beginning of the experiment. Each animal was housed separately throughout the experiment. Ten rats received septal lesions and the remaining 10 animals served as unoperated controls.

All animals were trained to barpress for $45-\mathrm{mg}$ food pellets in one of two standard BRS operant chambers with associated Digi-Bit Model 2901 programming units. The animals were allowed to obtain a minimum of 200 pellets immediately after acquisition of the barpressing habit. Following this, the animals were run in squads of five rats each in five Scientific Prototype operant chambers for 5 days on a continuous reinforcement (CRF) schedule. Subsequently, animals to be lesioned were operated on the sixth day and were then run another 5 days on the CRF schedule, beginning the day immediately following the lesion. Normal control animals were run on the CRF schedule for 5 additional days. Thus, both normal and lesioned animals received 10 days of training under the CRF schedule. Each daily run continued until each animal had obtained 150 reinforcements (approximately $10-20 \mathrm{~min}$ ). The animals were then placed on a DRL 20 schedule of reinforcement on the day following CRF training. On the DRL schedule the animals were required to wait at least $20 \mathrm{sec}$ between barpresses in order to receive reinforcement. The animals received 16 days of DRL 20 training. Each daily DRL run lasted $50 \mathrm{~min}$, after which each animal was allowed to consume approximately $10 \mathrm{~g}$ of a wet mash of Purina laboratory chow.

The Scientific Prototype operant chambers were linked to an IBM-1800 Data Acquisition and Control System, which programmed the runs and the reinforcement schedules and recorded responses for each animal. Responses were recorded as interresponse times (IRTs) and stored in an IBM-2310 disk
Behavioral Procedures storage unit until the end of each day's runs, when they were punched onto cards by an IBM-1442 card $\mathrm{read} / \mathrm{punch}$. The cards were later read back to disk, and IRTs were gated into 5 -sec bins. Total responses and reinforcements, as well as relative frequencies of different IRTs and response probabilities, were determined and printed, again utilizing the 1800 system and associated input/output devices.

Surgery and Histology

Septally lesioned animals received sodium pentobarbital (Nembutal) anesthesia ( $40 \mathrm{mg} / \mathrm{kg}$ body weight, IP), preceded by $0.5 \mathrm{cc}(0.1 \mathrm{mg})$ atropine sulfate to reduce respiratory distress. Local infiltration of xylocaine hydrochloride $(2 \%)$ with epinepherine was used during surgery to supplement the Nembutal anesthesia and to reduce bleeding. Each animal received $0.2 \mathrm{cc}$ procaine penicillin $\mathbf{G}(60,000$ units, Duracillin) postoperatively.

A midline incision was made, the skull exposed, and a hole drilled at the proper coordinate with a dental drill Anodal electrolytic lesions ( $2 \mathrm{~mA}$ for $20 \mathrm{sec}$ ) were made by a Stoelting 58040 electrolytic lesion maker, utilizing a Baltimore Instrument $\mathrm{Co}$. stereotaxic instrument for stereotaxic orientation. Lesion coordinates were taken from the rat brain atlas of Pellegrino \& Cushman (1967). Midline septal lesions were placed $7.8 \mathrm{~mm}$ anterior to and $6.5 \mathrm{~mm}$ above the interaural line.

Following completion of the experiment, the lesioned animals were sacrificed with a lethal dose of Nembutal, perfused intracardially with Ringer's saline solution, and the brains were fixed in formalin. Serial frozen sections, 50 microns thick, photographed as wet slides, were used for lesion localization.

RESULTS AND DISCUSSION

All septal lesions extended rather deeply and slightly involved the medial parolfactory area. All but two of the lesions involved the diagonal band of Broca. Slight intrusion into other neighboring tissue (with the number of animals involved) was as follows: fornix (5); hippocampal commissure (5); triangular septal nucleus (4); very slight, circumscribed damage to overlying corpus callosum and neocortex (3); nucleus proprious commissurae anterioris (2); and paratenial and paraventricular nuclej of the thalamus (1).

Figure 1 (A and B) presents mean responses emitted and reinforcements obtained for the normal and septal animals over the 16 days of DRL training. A two-factor analysis of variance (Lesions by Days, with repeated measures on days) was performed on responses and 
reinforcements (Winer, 1962). The septal animals responded significantly more $(F=33.92, \quad p<.01)$ and received significantly fewer reinforcements $(F=25.23, p<.01)$ than normal control animals. However, even though both septal and normal animals significantly reduced response rates over days $(F=11.03, p<.01)$, no corresponding change in reinforcement rate took place $(F=0.07)$. No significant interactions were found with respect to either responses emitted $(F=3.62)$ or reinforcements obtained $(F=0.03)$.

In contrast to the earlier reports of Ellen et al (1964) and MacDougall et al (1969), only the normal animals in the present study showed evidence of temporal discrimination, as measured by the IRTs/OP statistic (Fig. 2). Normal animals developed an increasing probability of responding as the delay between responses increased within the first few (5-6) days of training. Response probability attained its asymptotic level by about 10 days. Septal animals, on the other hand, developed a high and equal likelihood of responding for all values of delay between responses early in the course of training. This remained constant throughout the remainder of the experiment. Furthermore, despite the temporal discrimination shown by the normal animals, they received only about $20 \%$ of the number of reinforcements possible in the 50 -min session. Since reinforcement frequency did not increase over the 16 days of training, it would seem that the occurrence of temporal discrimination is not a sufficient condition for obtaining reinforcement on the DRL schedule.

Two findings in the present study differ from previously reported results (Ellen et al, 1964; MacDougall et al, 1969). First, septal animals failed to acquire a temporal discrimination. Second, while demonstrating a temporal discrimination, normal animals received relatively few reinforcements (approximately 30 in $50 \mathrm{~min}$ ) and did not increase their reinforcement frequency over 16 days of training. The basis for these differences is not immediately apparent. One possibility is that the animals in the previous studies were influenced to a certain degree by the sounds of the programming and recording equipment. In the earlier studies the programming and recording devices were either electromechanical and/or solid state. Despite the use of various combinations of sound-attenuated chambers, masking (e.g., ventilation fan) noises, and possibly the location of the programming and recording equipment in another (though adjacent) room,
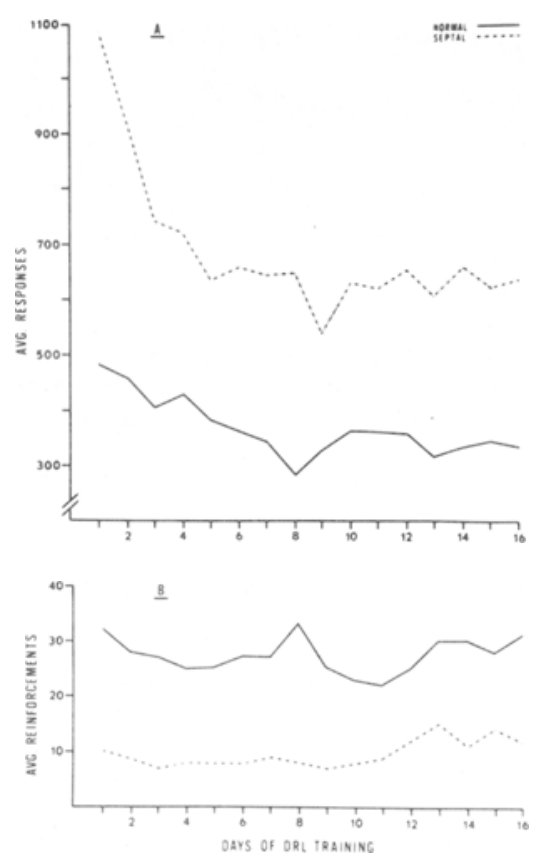

Fig. 1. Changes in response rate (A) and reinforcements obtained $(B)$ as a function of days of training.

noises associated with the operation of programming and recording devices can, to some variable degree, become correlated with the elapsing of the required delay on the DRL schedule. To the extent that this occurs, these noises will acquire cue functions and performance will come under their control. Ellen \& Butter (1969) have shown that even septal animals can obtain frequencies of reinforcement

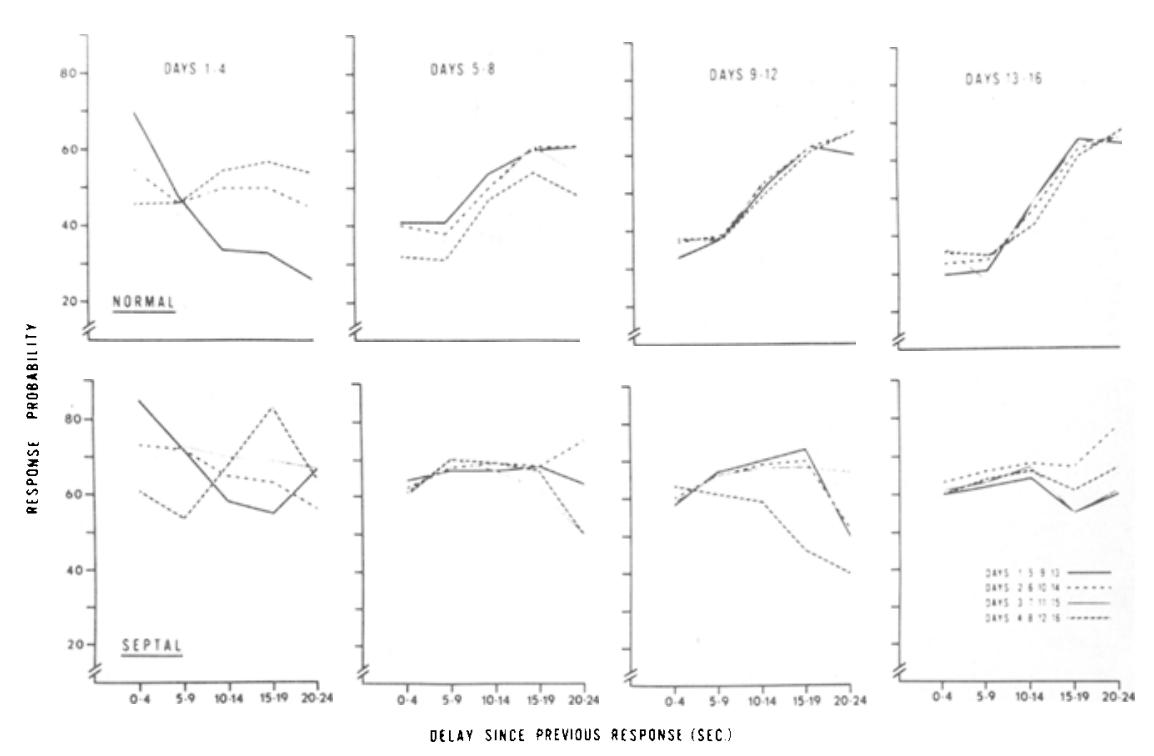

Fig. 2. Daily response probabilities (IRTs/OP) as a function of delay since a previous response. comparable to that of normal animals when provided with a definite consistent exteroceptive cue to signal the end of the required delay. In the present study, however, no such equipment noises were present, since the animals were run over $200 \mathrm{ft}$ and several walls distant from the computer used to program the reinforcement contingencies and record the responses. The differences between the results of the present study and previous ones would suggest that in the earlier studies the isolation of the animals from exteroceptive cueing was actually less effective than assumed.

One final question remains. To the extent that the absence of any exteroceptive cueing precluded both septals and normal rats from increasing their reinforcement rate as training on the schedule progressed, what accounts for the fact that the normal animals were able to acquire a temporal discrimination while the septals were not? In a recent article, Zuriff (1969) suggested that temporal discrimination reflects the discrimination or abstraction of duration as an invariant from the total flux of stimulation, either internal or external, that is present. Since no differential external stimulation was present in our situation and since reinforcement frequencies remained constant throughout the course of training, it seems reasonable to assume that differential internal effects resulted from nonreinforcement of responses of different delays, and that it is from these differential effects that duration must be abstracted. That is, nonreinforcement after a response

$$
\text { (1) }
$$


having an 18-sec delay produces eflects which are discriminably different from nonreinforcement after a response having a 2 -sec delay since the preceding response. However, in previous studies (Ellen \& Bate, 1969, 1970), we have shown that septal animals fail to utilize differential internal stimulation as discriminative cues for behavior. Thus, it would appear that the differential internal effects of nonreinforcement of responses having different delays since a previous response are not discriminated by the septal animals and thus no temporal discrimination is acquired. This hypothesis requires more precise specification as to the nature and properties of the internal stimulation which must be discriminated, while at the same time focusing attention upon the bases underlying the failure to discriminate differential effects of nonreinforcement.

\section{REFERENCES}

ANGER, D. Dependence of interresponse times upon the relative reinforcement of different interresponse times. Journal of Experimental Psycholog:, 1956,52 , 145-161.

ELLEN, P., \& BATE, G. Qualitative differences in the maze performance of rats with septal lesions. Pstchonomic Science, $1969,15,5-6$.

ELLEN, P.. \& BATE, G. Limbic lesions and cue utilization in the Dashiell maze. Psychonomic Science, 1970, 19, 47-49.

ELLEN, P., \& BUTTER, J. External cue control of DRL performance in rats with septal lesions. Physiology \& Beharior. $1969,4,1-6$

ELLEN, P WILSON, A, S, \& POWELL, E. W. Septal inhibition and timing behavior in the rat. Experimental Neurology, 1964. 10, $120-132$.

MACDOUGALL, J. M., VANHOESEN, G. W.. \& MITCHELL, J. C. Development of post $S R$ and post non $S R$ DRL performance and its retention following septal lesions in rats. Psychonomic Science, 1969, 16, 45-46.

PELLEGRINO L J \& CUSHMAN, A. J. A stereotaxic atlas of the rat brain. New York: Meredith, 1967.

WINER, B. J. Statistical principles in experimental design. New York: McGraw-Hill, 1962.

ZURIFF, G. E. Collateral responding during differential reinforcement of low rates. Journal of the Experimental Analysis of Behavior, 1969, 12, 971-976.

\section{Attention and conditioned suppression*}

\author{
C. J. BRIMER $\dagger$ \\ Dalhousie University, Halifax, N.S., Canada
}

Experimental rats were first trained to inhibit responding during a TO period that was preceded by a light signal. In subsequent standard CER training, a noise CS was paired with electric shock. When the experimental Ss were tested with the light/noise compound, their response rate during TO was not different from that of control Ss who had had no TO experience. The results were interpreted in terms of the suggestion that animals fail to attend to appetitive cues during conditioned suppression.

In the typical conditioned suppression or conditioned emotional response (CER) demonstration, the presentation of a signal (CS), which has been paired with electric shock (US), is observed to depress the rate of responding for appetitive reinforcement. Although no completely satisfactory explanation exists for the conditioned suppression phenomenon, most investigators have tended to identify the phenomenon with classical conditioning (Kamin, 1965; Lyon, 1968).

A number of years ago, Broadbent (1953) suggested that many of the features of classical conditioning could be interpreted in terms of an attention type of mechanism. Extrapolating this idea to the CER situation would suggest that conditioned suppression occurs as a result of a shift in the

* This research was supported by Grant APA-105 to the author from the National Research Council of Canada.

tThe author wishes to express his thanks to Mr. Ronald Van Houten and Mr. John Wright for experimental assistance. animal's focus of attention. When an aversive $C S$ is presented, the animal ceases to attend to the operant cues and, consequently, ceases to respond. The following experiment, which attempted to test such an idea, involved three main training phases. In the first phase, rats were given discrimination training with a preextinction stimulus. During the second phase, $S$ s received standard CER training. In the final phase, the Ss were tested with a compound stimulus composed of the preextinction and aversive signals. The prediction was that animals would fail to attend to the extinction cue during the compound test, as indexed by their postsignal behavior.

\section{METHOD}

The Ss were adult male hooded rats maintained at approximately $75 \%$ of ad lib weight. Out of an original group of 17,1 animal died during the course of the experiment and 3 other $\mathrm{Ss}$ had to be discarded due to experimental errors. Consequently, all of the analyses of the data were restricted to the surviving group of 13 Ss.
Animals in the main experimental condition were first trained to leverpress on a 30-sec VI food-reinforcement schedule. The Ss were then given discrimination training during which a 2 -min stimulus signaled a subsequent 6 -min timeout period (TO) during which experimental extinction was in effect. The preextinction signal was an overhead light which produced a level of illumination of approximately $2.14 \mathrm{fc}$, as measured at the rat's normal location in the animal chamber. No external stimulus was presented during the TO period itself. Three signal-extinction pairings were given during each 1 -h daily training session. This training had to be continued for approximately 3 months in order to obtain reliable postsignal suppression.

Following the discrimination experience, animals were given standard CER training. The CS was a $3-\mathrm{min} 70-\mathrm{dB}$ white noise that terminated with the delivery of a $1 / 2$-sec 1.0-mA electric shock. There were 4 days of CER training interspersed among the final 10 days of training. On each daily 1 -h session either three signaled TO or three CER trials were given. With the exception of the $\mathrm{TO}$ periods, the VI food-reinforcement schedule remained in effect throughout each training session.

On the compound test day, the preextinction signal was presented during the final $2 \mathrm{~min}$ of the noise CS. There were three compound trials administered during the 1 -h test session.

The main features of the experimental training conditions are presented in diagramatic form in Fig. 1.

During discrimination training, responses were recorded for the $2 \mathrm{~min}$ preceding each TO signal, for the $2 \mathrm{~min}$ of the signal, and for the three 2 -min periods of extinction. Similar records were obtained for the CER training and compound test phases of the experiment.

A "no-extinction" control group received the same training program as the experimental Ss, except that the VI reinforcement schedule remained in effect during the postlight period. The procedure for the "no-shock" group was identical to that of the experimental animals, except that shock was never given at the termination of the noise. There were seven experimental animals, four no-extinction, and two no-shock control Ss.

RESULTS AND DISCUSSION

A summary of the main results is presented in Table 1 . The ratio scores were calculated by comparing the response rates during the 2 -min signal (or for the post scores, the $2 \mathrm{~min}$ 